\title{
A new stability result for a thermoelastic Bresse system with viscoelastic damping
}

\section{Soh Edwin Mukiawa ${ }^{1 *}$ (D, Cyril Dennis Enyi ${ }^{1}$ and Tijani Abdulaziz Apalara}

\section{"Correspondence:}

mukiawa@uhb.edu.sa

'Department of Mathematics,

University of Hafr Al Batin, Hafr Al

Batin 31991, Saudi Arabia

\begin{abstract}
We investigate a thermoelastic Bresse system with viscoelastic damping acting on the shear force and heat conduction acting on the bending moment. We show that with weaker conditions on the relaxation function and physical parameters, the solution energy has general and optimal decay rates. Some examples are given to illustrate the findings.
\end{abstract}

MSC: 35B35; 35L05; 35B40; 35L20; 74D10; 93D15

Keywords: General decay; Fourier law; Bresse system; Viscoelastic law; Convexity

\section{Introduction}

The motion of the classical Bresse system without any external forces is described by the following system:

$$
\begin{cases}\rho_{1} u_{t t}-S_{x}-l N=0, & x \in(0, L), t>0 \\ \rho_{2} v_{t t}-M_{x}+S=0, & x \in(0, L), t>0 \\ \rho_{1} w_{t t}-N_{x}+l S=0, & x \in(0, L), t>0\end{cases}
$$

where $u=u(x, t)$ is the vertical angle displacement, $v=v(x, t)$ is the shear angle displacement, and $w=w(x, t)$ is the longitudinal angle displacement. The physical constants $\rho_{1}=\rho A, \rho_{2}=\rho I, l=R^{-1}$, where $\rho, A, I, R$ are the material density, cross sectional curvature, second moment of the cross-section, and radius of curvature, respectively. The constitutive laws denoted by $N, M$, and $S$ are respectively the axial force, the bending moment, and the shear force. System (1.1) has been investigated by many authors in the literature, and the results of well-posedness, stability (polynomial and exponential) have been established, see for example results in [1-13] and the references cited therein. Recently, Messauodi and Hassan [14] considered

$$
\begin{cases}\rho_{1} u_{t t}-k_{1}\left(u_{x}+v+l w\right)_{x}-k_{3} l\left(w_{x}-l u\right)=0, & x \in(0, L), t>0, \\ \rho_{2} v_{t t}-k_{2} v_{x x}+k_{1}\left(u_{x}+v+l w\right)-\int_{0}^{t} g(t-s) v_{x x}(x, s) d s=0, & x \in(0, L), t>0, \\ \rho_{1} w_{t t}-k_{3}\left(w_{x}-l u\right)_{x}+k_{1} l\left(u_{x}+v+l w\right)=0, & x \in(0, L), t>0,\end{cases}
$$

(c) The Author(s) 2021. This article is licensed under a Creative Commons Attribution 4.0 International License, which permits use sharing, adaptation, distribution and reproduction in any medium or format, as long as you give appropriate credit to the original author(s) and the source, provide a link to the Creative Commons licence, and indicate if changes were made. The images or other third party material in this article are included in the article's Creative Commons licence, unless indicated otherwise in a credit line to the material. If material is not included in the article's Creative Commons licence and your intended use is not permitted by statutory regulation or exceeds the permitted use, you will need to obtain permission directly from the copyright holder. To view a copy of this licence, visit http://creativecommons.org/licenses/by/4.0/. 
with Dirichlet-Neumann-Neumann boundary conditions and proved that the solution energy has a general decay rate if

$$
\frac{k_{1}}{\rho_{1}}=\frac{k_{2}}{\rho_{2}} \quad \text { and } \quad k_{1}=k_{3} \text {. }
$$

Furthermore, they established a weaker stability result for strong solution provided

$$
\frac{k_{1}}{\rho_{1}} \neq \frac{k_{2}}{\rho_{2}} \text { and } k_{1}=k_{3} \text {. }
$$

Now, considering the thermoelastic dissipation effect on the bending moment of a Bresse system, where the heat is given by Fourier's law, we have (see $[15,16])$

$$
\rho_{3} \theta_{t}+q_{x}+\gamma v_{x t}=0, \quad x \in(0, L), t>0,
$$

where $\theta=\theta(x, t)$ is the temperature difference, $q$ represents the heat flux, $\rho_{3}$ and $\gamma$ are capacity and adhesive stiffness, respectively. Coupling (1.1) and (1.5), we arrive at the thermoelastic Bresse system

$$
\begin{cases}\rho_{1} u_{t t}-S_{x}-l N=0, & x \in(0, L), t>0, \\ \rho_{2} v_{t t}-M_{x}+S=0, & x \in(0, L), t>0, \\ \rho_{1} w_{t t}-N_{x}+l S=0, & x \in(0, L), t>0, \\ \rho_{3} \theta_{t}+q_{x}+\gamma v_{x t}=0, & x \in(0, L), t>0 .\end{cases}
$$

For the physical and mathematical justification of (1.6), we refer to some constitutive laws of mathematical physics which connect the works of Timoshenko $[17,18]$ to the elastic or thermoelastic relations for a thin beam or plate which is assumed to be homogeneous and elastically or thermally isotropic, see $[15,16,19-23,29]$ and the references therein.

In the present work, with the viscoelastic law applied to the shear force, the following constitutive laws are imminent:

$$
\left\{\begin{array}{l}
S=k_{1}\left(u_{x}+v+l w\right)-\int_{0}^{t} g(t-s)\left(u_{x}+v+l w\right)(x, s) d s, \\
M=k_{2} v_{x}-\gamma \theta, \quad N=k_{3}\left(w_{x}-l u\right), \quad q=-\beta \theta_{x},
\end{array}\right.
$$

where $k_{1}=\kappa G A, k_{2}=E I, k_{3}=E A, E, G, \kappa$, and $\beta$ are the modulus of elasticity, shear modulus, shear factor, and diffusivity, respectively. The viscoelastic term (represented by the convolution, see $[24,25]$ for details) acts as a damping mechanism to decrease the effect of unwanted vibration from internal or external forces such as beam's weight and wind. Substituting (1.6) into (1.7), we arrive at the following Timoshenko-thermoelastic Bresse system:

$$
\left\{\begin{array}{l}
\rho_{1} u_{t t}-k_{1}\left(u_{x}+v+l w\right)_{x}-k_{3} l\left(w_{x}-l u\right)+\int_{0}^{t} g(t-s)\left(u_{x}+v+l w\right)_{x} d s=0, \\
\rho_{2} v_{t t}-k_{2} v_{x x}+k_{1}\left(u_{x}+v+l w\right)-\int_{0}^{t} g(t-s)\left(u_{x}+v+l w\right) d s+\gamma \theta_{x}=0, \\
\rho_{1} w_{t t}-k_{3}\left(w_{x}-l u\right)_{x}+k_{1} l\left(u_{x}+v+l w\right)-l \int_{0}^{t} g(t-s)\left(u_{x}+v+l w\right) d s=0, \\
\rho_{3} \theta_{t}-\beta \theta_{x x}+\gamma v_{x t}=0,
\end{array}\right.
$$


where $x \in(0, L), t>0$, the physical parameters $l, k_{1}, k_{2}, k_{3}, \rho_{1}, \rho_{2}, \rho_{3}, \beta, \gamma$ are all positive constants, and $g$ is a given function to be specified later. Recently, Mukiawa [26] considered (1.8) with Maxwell-Cattaneo law and established a general decay estimate. Next, we supplement (1.8) with the Neumann-Dirichlet-Dirichlet-Neumann boundary conditions:

$$
\begin{cases}u_{x}(0, t)=u_{x}(L, t)=v(0, t)=v(L, t)=0, & t \geq 0 \\ w(0, t)=w(L, t)=\theta_{x}(0, t)=\theta_{x}(L, t)=0, & t \geq 0\end{cases}
$$

and the initial data

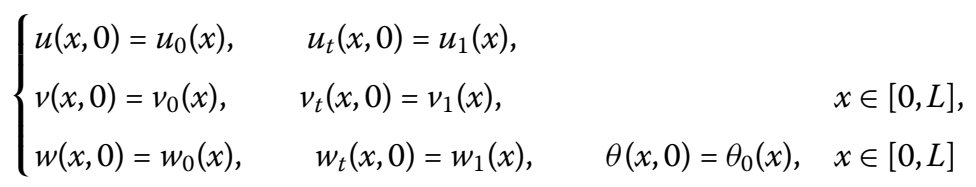

Our main focus is to show that the solution energy of (1.8)-(1.10) decays in the same way as the relaxation function $g$ provided $k_{1}=k_{3}$. Thus, without additional conditions on the physical parameters $k_{1}, \rho_{1}, k_{2}, \rho_{2}$ and without stronger regularity on the solution $(u, v, w, \theta)$ of system (1.8)-(1.10), we obtain a general and optimal decay estimate for the solution energy. The result obtained in this paper is general and optimal in the sense that it agrees with the decay rate of $g$ (see conditions on $g$ in Sect. 2). This work is organized as follows: In Sect. 2, we present materials that will be helpful in establishing the main results. In Sect. 3, we state and prove some useful lemmas. In Sect. 4, we look at the stability rate of the energy functional associated with problem (1.8)-(1.10).

\section{Assumptions and functional setting}

We denote by $\langle\cdot, \cdot\rangle$ and $\|\cdot\|_{2}$ the usual inner product and the norm in $L^{2}(0, L)$, respectively. In addition, we assume that the relaxation function $g$ satisfies

$\left(A_{1}\right) g:[0,+\infty) \longrightarrow(0,+\infty)$ is a decreasing $C^{1}$ - function such that

$$
g(0)>0, \quad k_{1}-\int_{0}^{\infty} g(s) d s=l_{0}>0
$$

$\left(A_{2}\right)$ There exist a nonincreasing $C^{1}$ - function $\xi:[0,+\infty) \rightarrow[0,+\infty)$ and a $C^{1}$ function

$$
H:[0,+\infty) \rightarrow[0,+\infty)
$$

that is linear or is strictly convex $C^{2}$ - function on $(0, r], r \leq g(0)$, with $H(0)=H^{\prime}(0)=$ 0 such that

$$
g^{\prime}(t) \leq-\xi(t) H(g(t)), \quad t \geq 0 .
$$

\section{Remark 2.1}

1. Condition $\left(A_{2}\right)$ implies there exists an extension $\bar{H}$ of $H$ such that

$$
\bar{H}:[0,+\infty) \rightarrow(0,+\infty)
$$


and $\bar{H}$ is a strictly increasing convex $C^{2}$-function. For example, for any $t>r$, we can define $\bar{H}$ by

$$
\bar{H}(\tau)=\frac{H^{\prime \prime}(r)}{2} \tau^{2}+\left(H^{\prime}(r)-H^{\prime \prime}(r) r\right) \tau+H(r)-H^{\prime}(r) r+\frac{H^{\prime \prime}(r)}{2} r^{2} .
$$

2. From $\left(A_{1}\right), g$ is continuous, positive and $g(0)>0$, hence for any $t_{0}>0$, we obtain

$$
\int_{0}^{t} g(s) d s \geq \int_{0}^{t_{0}} g(s) d s=g_{0}>0, \quad \forall t>t_{0} .
$$

3. Again, condition $\left(A_{2}\right)$ implies that $\xi$ and $g$ are continuous, nonincreasing, and positive. Moreover, $H$ is continuous and positive. Hence, $\forall t \in\left[0, t_{0}\right]$, we obtain

$$
0<g\left(t_{0}\right) \leq g(t) \leq g(0), \quad 0<\xi\left(t_{0}\right) \leq \xi(t) \leq \xi(0)
$$

which implies

$$
b_{1} \leq \xi(t) H(g(t)) \leq b_{2},
$$

where $b_{1}$ and $b_{2}$ are some positive constants. Hence, it follows that

$$
g^{\prime}(t) \leq-\xi(t) H(g(t)) \leq-\frac{b_{1}}{g(0)} g(0) \leq-\frac{b_{1}}{g(0)} g(t), \quad \forall t \in\left[0, t_{0}\right] .
$$

Remark 2.2 Let

$$
m(u)=\frac{1}{L} \int_{0}^{L} u(x, t) d x, \quad m(\theta)=\frac{1}{L} \int_{0}^{L} \theta(x, t) d x .
$$

Integration of $(1.8)_{1}$ and $(1.8)_{4}$ over $(0, L)$ yields

$$
\frac{d^{2}}{d t^{2}} m(u)+\frac{k_{3} l^{2}}{\rho_{1}} m(u)=0 \text { and } \quad \frac{d}{d t} m(\theta)=0 .
$$

Solving (2.8) and using the initial data $u_{0}, u_{1}$, and $\theta_{0}$ yield

$$
m(u)=B_{0} \cos \left(\alpha_{0} t\right)+B_{1} \sin \left(\alpha_{0} t\right) \quad \text { and } \quad m(\theta)=m\left(\theta_{0}\right)
$$

where

$$
\alpha_{0}=\sqrt{\frac{k_{3}}{\rho_{1}}} l, \quad B_{0}=\int_{0}^{L} u_{0}(x) d x, \quad B_{1}=\frac{1}{\alpha_{0}} \int_{0}^{L} u_{1}(x) d x .
$$

Now, we define

$$
\tilde{u}=u-m(u), \quad \tilde{\theta}=\theta-m(\theta) .
$$

It follows that

$$
\int_{0}^{L} \tilde{u}(x, t) d x=0 \quad \text { and } \quad \int_{0}^{L} \tilde{\theta}(x, t) d x=0, \quad \forall t \geq 0 .
$$


Therefore, we can use Poincaré on $u$ and $\theta$. Hence, due to (2.10), we have the Poincaré inequality

$$
\|\tilde{\theta}\|_{2}^{2} \leq c_{p}\left\|\theta_{x}\right\|_{2}^{2} .
$$

Moreover, $(\tilde{u}, v, w, \tilde{\theta})$ satisfies problem (1.8) with initial data for $\tilde{u}$ and $\tilde{\theta}$ given as

$$
\tilde{u}_{0}=u_{0}-m\left(u_{0}\right), \quad \tilde{u_{1}}=u_{1}-m\left(u_{1}\right) \quad \text { and } \quad \tilde{\theta}_{0}=\theta_{0}-m\left(\theta_{0}\right) .
$$

From now onward, we work with $(\tilde{u}, v, w, \tilde{\theta})$; however, for convenience, we write $(u, v, w, \theta)$ keeping in mind Remark (2.2). Let us define the following spaces:

$$
\begin{aligned}
& L_{*}^{2}=L_{*}^{2}(0, L)=\left\{f \in L^{2}(0, L): \int_{0}^{L} f(x) d x=0\right\}, \\
& H_{*}^{1}=H_{*}^{1}(0, L)=H^{1}(0,1) \cap L_{*}^{2}(0, L), \\
& H_{*}^{2}=H_{*}^{2}(0, L)=\left\{f \in H^{2}(0, L): f_{x}(0)=f_{x}(L)=0\right\} .
\end{aligned}
$$

The well-posedness of problem (1.8)-(1.10) is the following.

Theorem 2.1 Let $\left(u_{0}, u_{1}, v_{0}, v_{1}, w_{0}, w_{1}, \theta_{0}\right) \in H_{*}^{1} \times L_{*}^{2} \times H_{0}^{1} \times L^{2} \times H_{0}^{1} \times L^{2} \times H_{*}^{1}$ and condition $\left(A_{1}\right)$ hold. Then problem (1.8)-(1.10) has a global weak unique solution $(u, v, w, \theta)$ such that

$$
\begin{aligned}
& (u, v, w) \in C\left([0,+\infty), H_{*}^{1} \times H_{0}^{1} \times H_{0}^{1}\right) \cap C^{1}\left([0,+\infty), L_{*}^{2} \times L^{2} \times L^{2}\right), \\
& \theta \in C\left([0,+\infty), H_{*}^{1}\right) .
\end{aligned}
$$

Moreover, if

$$
\begin{aligned}
& \left(u_{0}, u_{1}, v_{0}, v_{1}, w_{0}, w_{1}, \theta_{0}\right) \\
& \quad \in H_{*}^{2} \cap H_{*}^{1} \times H_{*}^{1} \times H^{2} \cap H_{0}^{1} \times H_{0}^{1} \times H^{2} \cap H_{0}^{1} \times H_{0}^{1} \times H_{*}^{2} \cap H_{*}^{1},
\end{aligned}
$$

then the weak unique solution of problem (1.8)-(1.10) has more regularity in the class

$$
\begin{aligned}
& u \in C\left([0,+\infty), H_{*}^{2} \cap H_{*}^{1}\right) \cap C^{1}\left([0,+\infty), H_{*}^{1}\right) \cap C^{2}\left((0,+\infty), L_{*}^{2}\right), \\
& v \in C\left([0,+\infty), H^{2} \cap H_{0}^{1}\right) \cap C^{1}\left([0,+\infty), H_{0}^{1}\right) \cap C^{2}\left((0,+\infty), L^{2}\right), \\
& w \in C\left((0,+\infty), H^{2} \cap H_{0}^{1}\right) \cap C^{1}\left([0,+\infty), H_{0}^{1}\right) \cap C^{2}\left((0,+\infty), L^{2}\right), \\
& \theta \in C\left((0,+\infty), H_{*}^{2} \cap H_{*}^{1}\right) \cap C^{1}\left([0,+\infty), H_{*}^{1}\right) .
\end{aligned}
$$

The result in Theorem 2.1 can be established using the Galerkin approximation method. The following notations and basic lemmas will be applied repeatedly throughout the paper. We set

$$
\begin{aligned}
& (g \circ f)(x, s, t)=\int_{0}^{t} g(t-s)(f(x, t)-f(x, s)) d s, \\
& (g \diamond f)(t)=\int_{0}^{t} g(t-s)\|f(t)-f(s)\|_{2}^{2} d s .
\end{aligned}
$$


Lemma 2.1 For any function $f \in L_{l o c}^{2}\left([0,+\infty), L^{2}(0,1)\right)$, we have

$$
\begin{aligned}
& \int_{0}^{L}\left(\int_{0}^{t} g(t-s)(f(t)-f(s)) d s\right)^{2} d x \leq\left(1-l_{0}\right)(g \diamond f)(t), \\
& \int_{0}^{L}\left(\int_{0}^{x} f(y, t) d y\right)^{2} d x \leq\|f(t)\|_{2}^{2} .
\end{aligned}
$$

For any $0<\alpha<1$, we set as in [27]

$$
h(t)=\alpha g(t)-g^{\prime}(t) \quad \text { and } \quad A_{\alpha}=\int_{0}^{+\infty} \frac{g^{2}(s)}{\alpha g(s)-g^{\prime}(s)} d s .
$$

Lemma 2.2 Let $(u, v, w, \theta)$ be the solution of problem (1.8)-(1.10). Then, for any $0<\alpha<1$, we have

$$
\begin{aligned}
& \int_{0}^{L}\left(\int_{0}^{t} g(t-s)\left(\left(u_{x}+v+l w\right)(t)-\left(u_{x}+v+l w\right)(s)\right) d s\right)^{2} d x \\
& \quad \leq A_{\alpha}\left(h \diamond\left(u_{x}+v+l w\right)\right)(t),
\end{aligned}
$$

where

$$
\left(h \diamond\left(u_{x}+v+l w\right)\right)(t)=\int_{0}^{t} h(t-s)\left\|\left(u_{x}+v+l w\right)(t)-\left(u_{x}+v+l w\right)(s)\right\|_{2}^{2} d s .
$$

Proof Using the Cauchy-Schwarz inequality, we have

$$
\begin{aligned}
& \int_{0}^{1}\left(\int_{0}^{t} g(t-s)\left(\left(u_{x}+v+l w\right)(t)-\left(u_{x}+v+l w\right)(s)\right) d s\right)^{2} d x \\
& =\int_{0}^{1}\left(\int_{0}^{t} \frac{g(t-s)}{\sqrt{h(t-s)}} \sqrt{h(t-s)}\left(\left(u_{x}+v+l w\right)(t)-\left(u_{x}+v+l w\right)(s)\right) d s\right)^{2} d x \\
& \leq\left(\int_{0}^{+\infty} \frac{g^{2}(s)}{h(s)} d s\right) \int_{0}^{1} \int_{0}^{t} h(t-s)\left(\left(u_{x}+v+l w\right)(t)-\left(u_{x}+v+l w\right)(s)\right)^{2} d s d x \\
& =\left(\int_{0}^{+\infty} \frac{g^{2}(s)}{\alpha g(s)-g^{\prime}(s)} d s\right)\left(h \diamond\left(u_{x}+v+l w\right)\right)(t) .
\end{aligned}
$$

Lemma 2.3 Let $F$ be a convex function on the close interval $[a, b], f: \Omega \rightarrow[a, b]$ and $j$ be an integrable function on $\Omega$ such that $j(x) \geq 0$ and $\int_{\Omega} j(x) d x=\beta_{1}>0$. Then we have the following Jensen inequality:

$$
F\left(\frac{1}{\beta_{1}} \int_{\Omega} f(y) j(y) d y\right) \leq \frac{1}{\beta_{1}} \int_{\Omega} F(f(y)) j(y) d y .
$$

\section{Technical lemmas}

In this section, we establish some lemmas which will be used to prove the main stability result. 
Lemma 3.1 Let $(u, v, w, \theta)$ be the solution of (1.8)-(1.10). Then

$$
\frac{d}{d t} E(t)=-\frac{1}{2} g(t)\left\|u_{x}+v+l w\right\|_{2}^{2}+\frac{1}{2}\left(g^{\prime} \diamond\left(u_{x}+v+l w\right)\right)(t)-\beta\left\|\theta_{x}\right\|_{2}^{2} \leq 0, \quad \forall t \geq 0,
$$

where

$$
\begin{aligned}
E(t)= & \frac{1}{2}\left(\rho_{1}\left\|u_{t}\right\|_{2}^{2}+\rho_{2}\left\|v_{t}\right\|_{2}^{2}+\rho_{1}\left\|w_{t}\right\|_{2}^{2}+\left(k_{1}-\int_{0}^{t} g(s) d s\right)\left\|u_{x}+v+l w\right\|_{2}^{2}\right) \\
& +\frac{k_{2}}{2}\left\|v_{x}\right\|_{2}^{2}+\frac{\rho_{3}}{2}\|\theta\|_{2}^{2}+\frac{k_{3}}{2}\left\|w_{x}-l u\right\|_{2}^{2}+\frac{1}{2}\left(g \diamond\left(u_{x}+v+l w\right)\right)(t) .
\end{aligned}
$$

Proof Multiplying the equations in (1.8) by $u_{t}, v_{t}, w_{t}$, and $\theta$, respectively, then integrating by parts over $(0, L)$ and using the boundary conditions, we get respectively

$$
\begin{aligned}
& \frac{1}{2} \frac{d}{d t} \rho_{1}\left\|u_{t}\right\|_{2}^{2}+\frac{1}{2} \frac{d}{d t} k_{1}\left\|u_{x}+v+l w\right\|_{2}^{2}-k_{1} \int_{0}^{L}\left(u_{x}+v+l w\right) v_{t} d x \\
& \quad-k_{1} l \int_{0}^{L}\left(u_{x}+v+l w\right) w_{t} d x-k_{3} l \int_{0}^{L}\left(w_{x}-l u\right) u_{t} d x \\
& \quad-\int_{0}^{L}\left(u_{x}+v+l w\right)_{t} \int_{0}^{t} g(t-s)\left(u_{x}+v+l w\right)(x, s) d s d x \\
& \quad+\int_{0}^{L} v_{t} \int_{0}^{t} g(t-s)\left(u_{x}+v+l w\right)(x, s) d s d x \\
& \quad+l \int_{0}^{L} w_{t} \int_{0}^{t} g(t-s)\left(u_{x}+v+l w\right)(x, s) d s d x=0, \\
& \frac{1}{2} \frac{d}{d t} \rho_{2}\left\|v_{t}\right\|_{2}^{2}+\frac{1}{2} \frac{d}{d t} k_{2}\left\|v_{x}\right\|_{2}^{2}+k_{1} \int_{0}^{L}\left(u_{x}+v+l w\right) v_{t} d x \\
& \quad-\int_{0}^{L} v_{t} \int_{0}^{t} g(t-s)\left(u_{x}+v+l w\right)(x, s) d s d x+\gamma \int_{0}^{L} \theta_{x} v_{t} d x=0, \\
& \frac{1}{2} \frac{d}{d t} \rho_{1}\left\|w_{t}\right\|_{2}^{2}+\frac{1}{2} \frac{d}{d t} k_{3}\left\|w_{x}-l u\right\|_{2}^{2}+k_{3} l \int_{0}^{L}\left(w_{x}-l u\right) u_{t} d x \\
& \quad+k_{1} l \int_{0}^{L}\left(u_{x}+v+l w\right) w_{t} d x-l \int_{0}^{L} w_{t} \int_{0}^{t} g(t-s)\left(u_{x}+v+l w\right)(x, s) d s d x=0
\end{aligned}
$$

and

$$
\frac{\rho_{3}}{2} \frac{d}{d t}\|\theta\|_{2}^{2}+\beta\left\|\theta_{x}\right\|_{2}^{2}-\gamma \int_{0}^{L} \theta_{x} v_{t} d x=0
$$

Adding (3.3)-(3.6) yields

$$
\begin{aligned}
\frac{1}{2} & \frac{d}{d t}\left(\rho_{1}\left\|u_{t}\right\|_{2}^{2}+\rho_{2}\left\|v_{t}\right\|_{2}^{2}+\rho_{1}\left\|w_{t}\right\|_{2}^{2}+k_{1}\left\|u_{x}+v+l w\right\|_{2}^{2}\right) \\
& +\frac{1}{2} \frac{d}{d t}\left(k_{2}\left\|v_{x}\right\|_{2}^{2}+k_{3}\left\|w_{x}-l u\right\|_{2}^{2}+\rho_{3}\|\theta\|_{2}^{2}\right) \\
& \underbrace{-\int_{0}^{L}\left(u_{x}+v+l w\right)_{t} \int_{0}^{t} g(t-s)\left(u_{x}+v+l w\right)(x, s) d s d x}_{J_{1}}=-\beta\left\|\theta_{x}\right\|_{2}^{2} .
\end{aligned}
$$


We estimate $J_{1}$ as follows:

$$
\begin{aligned}
J_{1}= & \int_{0}^{L}\left(u_{x}+v+l w\right)_{t} \int_{0}^{t} g(t-s)\left(\left(u_{x}+v+l w\right)(t)-\left(u_{x}+v+l w\right)(s)\right) d s d x \\
& -\int_{0}^{t} g(s) d s \int_{0}^{L}\left(u_{x}+v+l w\right)_{t}\left(u_{x}+v+l w\right) d x \\
= & \frac{1}{2} \int_{0}^{1} \int_{0}^{t} g(t-s) \frac{d}{d t}\left(\left(u_{x}+v+l w\right)(x, t)-\left(u_{x}+v+l w\right)(x, s)\right)^{2} d s d x \\
& -\frac{1}{2} \int_{0}^{t} g(s) d s \frac{d}{d t}\left\|u_{x}+v+l w\right\|_{2}^{2} \\
= & \frac{1}{2} \frac{d}{d t}\left(g \diamond\left(u_{x}+v+l w\right)\right)(t)-\frac{1}{2}\left(g^{\prime} \diamond\left(u_{x}+v+l w\right)\right)(t) \\
& -\frac{1}{2} \frac{d}{d t}\left(\left(\int_{0}^{t} g(s) d s\right)\left\|u_{x}+v+l w\right\|_{2}^{2}\right)+\frac{1}{2} g(t)\left\|u_{x}+v+l w\right\|_{2}^{2} .
\end{aligned}
$$

Inserting (3.8) into (3.7), we obtain (3.1). Hence, $E$ is decreasing and bounded above by $E(0)$.

Lemma 3.2 Suppose that $(u, v, w, \theta)$ is the solution of (1.8)-(1.10). Then the functional $F_{1}$ defined by

$$
F_{1}(t)=-\rho_{1} \int_{0}^{L}\left(w_{x}-l u\right) \int_{0}^{x} w_{t}(y, t) d y d x
$$

satisfies, for any positive $\epsilon_{0}$, the estimate

$$
\begin{aligned}
F_{1}^{\prime}(t) \leq & -\frac{k_{3}}{2}\left\|w_{x}-l u\right\|_{2}^{2}+\epsilon_{0}\left\|u_{t}\right\|_{2}^{2}+\left(\rho_{1}+\frac{\left(\rho_{1} l\right)^{2}}{4 \epsilon_{0}}\right)\left\|w_{t}\right\|_{2}^{2} \\
& +C l\left\|u_{x}+v+l w\right\|_{2}^{2}+C A_{\alpha}\left(h \diamond\left(u_{x}+v+l w\right)\right)(t), \quad \forall t \geq 0 .
\end{aligned}
$$

Proof Differentiation of $F_{1}$, using $(1.8)_{3}$, integration by parts, and the boundary conditions give

$$
\begin{aligned}
F_{1}^{\prime}(t)= & -\rho_{1} \int_{0}^{L}\left(w_{x}-l u\right)_{t} \int_{0}^{x} w_{t}(y, t) d y d x-\rho_{1} \int_{0}^{L}\left(w_{x}-l u\right) \int_{0}^{x} w_{t t}(y, t) d y d x \\
= & \rho_{1}\left\|w_{t}\right\|_{2}^{2}-k_{3}\left\|w_{x}-l u\right\|_{2}^{2}+\underbrace{\rho_{1} l \int_{0}^{L} u_{t} \int_{0}^{x} w_{t}(y, t) d y d x}_{J_{2}} \\
& +\underbrace{k_{1} l \int_{0}^{L}\left(w_{x}-l u\right) \int_{0}^{x}\left(u_{y}+v+l w\right)(y, t) d y d x}_{J_{3}} \\
& -\underbrace{l \int_{0}^{L}\left(w_{x}-l u\right) \int_{0}^{x} \int_{0}^{t} g(t-s)\left(u_{y}+v+l w\right)(y, s) d s d y d x}_{J_{4}} .
\end{aligned}
$$


Applying the Cauchy-Schwarz, Young inequalities and Lemmas 2.1-2.2, we estimate $J_{2}-$ $J_{4}$. We have, for any positive $\delta_{1}, \epsilon_{0}$,

$$
\begin{aligned}
J_{2} \leq & \epsilon_{0}\left\|u_{t}\right\|_{2}^{2}+\frac{\left(\rho_{1} l\right)^{2}}{4 \epsilon_{0}}\left\|w_{t}\right\|_{2}^{2}, \\
J_{3} \leq & \frac{k_{3} \delta_{0}}{2}\left\|w_{x}-l u\right\|_{2}^{2}+\frac{\left(k_{1} l\right)^{2}}{2 k_{3}^{2} \delta_{0}}\left\|u_{x}+v+l w\right\|_{2}^{2}, \\
J_{4} \leq & \frac{k_{3} \delta_{0}}{2}\left\|w_{x}-l u\right\|_{2}^{2}+\frac{\left(l_{0} l\right)^{2}}{k_{3}^{2} \delta_{0}}\left\|u_{x}+v+l w\right\|_{2}^{2} \\
& +\frac{C A_{\alpha}}{\delta_{0}}\left(h \diamond\left(u_{x}+v+l w\right)\right)(t) .
\end{aligned}
$$

Hence, (3.9) follows by substituting (3.11) into (3.10) and selecting $\delta_{0}=\frac{1}{2}$.

Lemma 3.3 Let $(u, v, w, \theta)$ be the solution of (1.8)-(1.10). Then the functional $F_{2}$ defined by

$$
F_{2}(t)=-\rho_{1} \int_{0}^{L}\left(u_{x}+v+l w\right) \int_{0}^{x} u_{t}(y, t) d y d x
$$

satisfies, for any positive $\epsilon_{1}, \epsilon_{2}$, the estimate

$$
\begin{aligned}
F_{2}^{\prime}(t) \leq & -\frac{l_{0}}{2}\left\|u_{x}+v+l w\right\|_{2}^{2}+\left(\rho_{1}+\frac{\rho_{1}^{2}}{4 \epsilon_{1}}+\frac{\left(\rho_{1} l\right)^{2}}{4 \epsilon_{2}}\right)\left\|u_{t}\right\|_{2}^{2}+\epsilon_{1}\left\|v_{t}\right\|_{2}^{2} \\
& +\epsilon_{2}\left\|w_{t}\right\|_{2}^{2}+C l\left\|w_{x}-l u\right\|_{2}^{2}+C A_{\alpha}\left(h \diamond\left(u_{x}+v+l w\right)\right)(t), \quad \forall t \geq 0 .
\end{aligned}
$$

Proof By direct differentiation, then using $(1.8)_{1}$, integration by parts, boundary condition (1.9) and keeping in mind (2.13), we get

$$
\begin{aligned}
F_{2}^{\prime}(t)= & \rho_{1}\left\|u_{t}\right\|_{2}^{2}-\underbrace{\rho_{1} \int_{0}^{L} v_{t} \int_{0}^{x} u_{t}(y, t) d y d x}_{J_{5}}-\underbrace{\rho_{1} l \int_{0}^{L} w_{t} \int_{0}^{x} u_{t}(y, t) d y d x}_{J_{6}} \\
& -\left(k_{1}-\int_{0}^{t} g(s) d s\right)\left\|u_{x}+v+l w\right\|_{2}^{2} \\
& -\underbrace{k_{3} l \int_{0}^{L}\left(u_{x}+v+l w\right) \int_{0}^{x}\left(w_{y}-l u\right)(y, t) d y d x}_{J_{7}} \\
& -\underbrace{\int_{0}^{L}\left(u_{x}+v+l w\right)\left(g \circ\left(u_{x}+v+l w\right)\right)(x, s, t) d x}_{J_{8}} .
\end{aligned}
$$


Using the Cauchy-Schwarz, Young inequalities and applying Lemmas 2.1-2.2, we have

$$
\begin{aligned}
& J_{5} \leq \epsilon_{1}\left\|v_{t}\right\|_{2}^{2}+\frac{\rho_{1}^{2}}{4 \epsilon_{1}}\left\|u_{t}\right\|_{2}^{2}, \\
& J_{6} \leq \epsilon_{2}\left\|w_{t}\right\|_{2}^{2}+\frac{\left(\rho_{1} l\right)^{2}}{4 \epsilon_{2}}\left\|u_{t}\right\|_{2}^{2}, \\
& J_{7} \leq \frac{\delta_{1}}{2}\left\|u_{x}+v+l w\right\|_{2}^{2}+\frac{\left(k_{3} l\right)^{2}}{2 \delta_{1}}\left\|w_{x}-l u\right\|_{2}^{2}, \\
& J_{8} \leq \frac{\delta_{1}}{2}\left\|u_{x}+v+l w\right\|_{2}^{2}+\frac{A_{\alpha}}{2 \delta_{1}}\left(h \diamond\left(u_{x}+v+l w\right)\right)(t) .
\end{aligned}
$$

Now, substituting (3.14) into (3.13), using the fact that $\left(k_{1}-\int_{0}^{t} g(s) d s\right) \geq l_{0}$, and choosing $\delta_{1}=\frac{l_{0}}{2}$, we obtain the result.

Lemma 3.4 The functional $F_{3}$ defined by

$$
F_{3}(t)=-\rho_{1} \int_{0}^{L} u_{t} \int_{0}^{x} \int_{0}^{t} g(t-s)\left(\left(u_{y}+v+l w\right)(y, t)-\left(u_{y}+v+l w\right)(y, s)\right) d s d y d x
$$

satisfies, for any positive $\epsilon_{3}, \epsilon_{4}$ and $t_{0}>0$, along the solution of (1.8)-(1.10), the estimate

$$
\begin{aligned}
F_{3}^{\prime}(t) \leq & -\frac{\rho_{1} g_{0}}{2}\left\|u_{t}\right\|_{2}^{2}+C\left\|v_{t}\right\|_{2}^{2}+C l^{2}\left\|w_{t}\right\|_{2}^{2}+\epsilon_{3}\left\|u_{x}+v+l w\right\|_{2}^{2}+\epsilon_{4}\left\|w_{x}-l u\right\|_{2}^{2} \\
& +C A_{\alpha}\left(1+\frac{1}{\epsilon_{3}}+\frac{1}{\epsilon_{4}}\right)\left(h \diamond\left(u_{x}+v+l w\right)\right)(t) .
\end{aligned}
$$

Proof Differentiating $F_{3}$, we obtain

$$
\begin{aligned}
F_{3}^{\prime}(t)= & -\underbrace{\rho_{1} \int_{0}^{L} u_{t} \int_{0}^{x} \int_{0}^{t} g^{\prime}(t-s)\left(\left(u_{y}+v+l w\right)(y, t)-\left(u_{y}+v+l w\right)(y, s)\right) d s d y d x}_{J_{9}} \\
& -\underbrace{\rho_{1} \int_{0}^{L} u_{t} \int_{0}^{x} \int_{0}^{t} g(t-s)\left(u_{y}+v+l w\right)_{t}(y, t) d s d y d x}_{J_{10}} \\
& -\underbrace{\rho_{1} \int_{0}^{L} u_{t t} \int_{0}^{x} \int_{0}^{t} g(t-s)\left(\left(u_{y}+v+l w\right)(y, t)-\left(u_{y}+v+l w\right)(y, s)\right) d s d y d x}_{J_{11}} .
\end{aligned}
$$

For $J_{9}$, we apply the Cauchy-Schwarz and Young inequalities, then recall that $h=\alpha g-g^{\prime}$, and applying Lemmas 2.1-2.2, we get, for any positive $\delta_{2}$,

$$
\begin{aligned}
J_{9}= & -\rho_{1} \int_{0}^{L} u_{t} \int_{0}^{x} \int_{0}^{t} h(t-s)\left(\left(u_{y}+v+l w\right)(y, t)-\left(u_{y}+v+l w\right)(y, s)\right) d s d y d x \\
& -\rho_{1} \alpha \int_{0}^{L} u_{t} \int_{0}^{x} \int_{0}^{t} g(t-s)\left(\left(u_{y}+v+l w\right)(y, t)-\left(u_{y}+v+l w\right)(y, s)\right) d s d y d x
\end{aligned}
$$




$$
\begin{aligned}
\leq & \frac{\delta_{2}}{2}\left\|u_{t}\right\|_{2}^{2}+\frac{C \rho_{1}^{2}}{\delta_{2}} \int_{0}^{L}\left(\int_{0}^{x} \int_{0}^{t} h(t-s)\left(\left(u_{y}+v+l w\right)-\left(u_{y}+v+l w\right)\right) d s d y\right)^{2} d x \\
& +\frac{C\left(\rho_{1} \alpha\right)^{2}}{\delta_{2}} \int_{0}^{L}\left(\int_{0}^{x} \int_{0}^{t} g(t-s)\left(\left(u_{y}+v+l w\right)-\left(u_{y}+v+l w\right)\right) d s d y\right)^{2} d x \\
\leq & \frac{\delta_{2}}{2}\left\|u_{t}\right\|_{2}^{2}+\frac{C \rho_{1}^{2}}{\delta_{2}} \int_{0}^{L} \int_{0}^{x}\left(\int_{0}^{t} h(t-s)\left(\left(u_{y}+v+l w\right)-\left(u_{y}+v+l w\right)\right) d s\right)^{2} d y d x \\
& +\frac{C\left(\rho_{1} \alpha\right)^{2}}{\delta_{2}} \int_{0}^{L} \int_{0}^{x}\left(\int_{0}^{t} g(t-s)\left(\left(u_{y}+v+l w\right)-\left(u_{y}+v+l w\right)\right) d s\right)^{2} d y d x \\
\leq & \frac{\delta_{2}}{2}\left\|u_{t}\right\|_{2}^{2}+\frac{C \rho_{1}^{2} L}{\delta_{2}} \int_{0}^{L}\left(\int_{0}^{t} h(t-s)\left(\left(u_{x}+v+l w\right)-\left(u_{x}+v+l w\right)\right) d s\right)^{2} d x \\
& +\frac{C\left(\rho_{1} \alpha\right)^{2} L}{\delta_{2}} \int_{0}^{L}\left(\int_{0}^{t} g(t-s)\left(\left(u_{x}+v+l w\right)-\left(u_{x}+v+l w\right)\right) d s\right)^{2} d x \\
\leq & \frac{\delta_{2}}{2}\left\|u_{t}\right\|_{2}^{2}+\left(\frac{C \rho_{1}^{2} L}{\delta_{2}}+\frac{C\left(\rho_{1} \alpha\right)^{2} L A_{\alpha}}{\delta_{2}}\right)\left(h \diamond\left(u_{x}+v+l w\right)\right)(t)
\end{aligned}
$$

For $J_{10}$, recalling (2.4) and (2.10), we have for any positive $\delta_{2}$

$$
\begin{aligned}
J_{10}= & -\rho_{1} \int_{0}^{L} u_{t} \int_{0}^{x} \int_{0}^{t} g(t-s)\left(u_{y}+v+l w\right)_{t}(y, t) d s d y d x \\
= & -\rho_{1}\left(\int_{0}^{t} g(s) d s\right) \int_{0}^{L} u_{t} \int_{0}^{x}\left(u_{y}+v+l w\right)_{t}(y, t) d y d x \\
= & -\rho_{1}\left(\int_{0}^{t} g(s) d s\right) \int_{0}^{L} u_{t} \int_{0}^{x} u_{y t}(y, t) d y d x \\
& -\rho_{1} \int_{0}^{t} g(s) d s \int_{0}^{L} u_{t} \int_{0}^{x} v_{t}(y, t) d y d x \\
& -\rho_{1} l\left(\int_{0}^{t} g(s) d s\right) \int_{0}^{L} u_{t} \int_{0}^{x} w_{t}(y, t) d y d x \\
= & -\rho_{1}\left(\int_{0}^{t} g(s) d s\right)\left\|u_{t}\right\|_{2}^{2}-\rho_{1} \int_{0}^{t} g(s) d s \int_{0}^{1} u_{t} \int_{0}^{x} v_{t}(y, t) d y d x \\
& -\rho_{1} l\left(\int_{0}^{t} g(s) d s\right) \int_{0}^{L} u_{t} \int_{0}^{x} w_{t}(y, t) d y d x \\
\leq & -\rho_{1} g_{0}\left\|u_{t}\right\|_{2}^{2}+\frac{\delta_{2}}{2}\left\|u_{t}\right\|_{2}^{2}+\frac{C\left(\rho_{1} g_{0}\right)^{2}}{\delta_{2}}\left\|v_{t}\right\|_{2}^{2}+\frac{C\left(\rho_{1} l g_{0}\right)^{2}}{\delta_{2}}\left\|w_{t}\right\|_{2}^{2} .
\end{aligned}
$$

Using (1.8) $)_{1}$, integration by parts, boundary conditions and recalling notation (2.13), we have

$$
\begin{aligned}
J_{11}= & k_{1} \int_{0}^{L}\left(u_{x}+v+l w\right)\left(g \circ\left(u_{x}+v+l w\right)\right)(x, s, t) d s d x \\
& -k_{3} l \int_{0}^{L}\left(w_{x}-l u\right) \int_{0}^{x}\left(g \circ\left(u_{y}+v+l w\right)\right)(y, s, t) d s d y d x \\
& -\int_{0}^{L}\left(\int_{0}^{t} g(t-s)\left(u_{x}+v+l w\right)(x, s) d s\right)\left(g \circ\left(u_{x}+v+l w\right)\right)(x, s, t) d x .
\end{aligned}
$$


Applying the Young inequality and Lemmas 2.1-2.2, we have, for any positive $\epsilon_{3}, \epsilon_{4}$,

$$
\begin{aligned}
J_{11} \leq & \frac{\epsilon_{3}}{2}\left\|u_{x}+v+l w\right\|_{2}^{2}+\frac{k_{1}^{2} A_{\alpha}}{2 \epsilon_{3}}\left(h \diamond\left(u_{x}+v+l w\right)\right)(t)+\epsilon_{4}\left\|w_{x}-l u\right\|_{2}^{2} \\
& +\frac{L\left(k_{3} l\right)^{2} A_{\alpha}}{4 \epsilon_{4}}\left(h \diamond\left(u_{x}+v+l w\right)\right)(t)+k_{1} A_{\alpha}\left(h \diamond\left(u_{x}+v+l w\right)\right)(t) \\
& +\frac{\epsilon_{3}}{2}\left\|u_{x}+v+l w\right\|_{2}^{2}+\frac{\left(l_{0}\right)^{2} A_{\alpha}}{2 \epsilon_{3}}\left(h \diamond\left(u_{x}+v+l w\right)\right)(t) .
\end{aligned}
$$

Substitution of (3.17)-(3.19) into (3.16) and selecting $\delta_{2}=\frac{\rho_{1} g_{0}}{2}$ yield (3.15). This completes the proof.

Lemma 3.5 Let $(u, v, w, \theta)$ be the solution of (1.8)-(1.10). Then the functional $F_{4}$ defined by

$$
F_{4}(t)=\rho_{2} \int_{0}^{L} v v_{t} d x
$$

satisfies the estimate

$$
\begin{aligned}
F_{4}^{\prime}(t) \leq & -\frac{k_{2}}{2}\left\|v_{x}\right\|_{2}^{2}+\rho_{2}\left\|v_{t}\right\|_{2}^{2}+C\left\|u_{x}+v+l w\right\|_{2}^{2} \\
& +C A_{\alpha}\left(h \diamond\left(u_{x}+v+l w\right)\right)(t)+C\left\|\theta_{x}\right\|_{2}^{2}, \quad \forall t \geq 0 .
\end{aligned}
$$

Proof Differentiating $F_{4}$, using $(1.8)_{2}$ and integration by parts yield

$$
\begin{aligned}
F_{4}^{\prime}(t)= & \rho_{2}\left\|v_{t}\right\|_{2}^{2}-k_{2}\left\|v_{x}\right\|_{2}^{2}-\underbrace{k_{1} \int_{0}^{L} v\left(u_{x}+v+l w\right) d x}_{J_{12}} \\
& +\underbrace{\int_{0}^{L} v \int_{0}^{t} g(t-s)\left(u_{x}+v+l w\right)(x, s) d s d x}_{J_{13}}-\underbrace{\gamma \int_{0}^{L} v \theta_{x} d x}_{J_{14}} .
\end{aligned}
$$

Applying the Young, Poincaré inequalities, Lemmas 2.1-2.2 and recalling (2.13), we obtain for any $\delta_{3}>0$

$$
\begin{aligned}
J_{12} \leq & \frac{\delta_{3} c_{p}}{4}\left\|v_{x}\right\|_{2}^{2}+\frac{C k_{1}^{2}}{\delta_{3}}\left\|u_{x}+v+l w\right\|_{2}^{2}, \\
J_{13}= & \int_{0}^{t} g(s) d s \int_{0}^{L} v\left(u_{x}+v+l w\right) d x-\int_{0}^{L} v\left(g \circ\left(u_{x}+v+l w\right)\right)(x, s, t) d x \\
\leq & \frac{\delta_{3} c_{p}}{4}\left\|v_{x}\right\|_{2}^{2}+\frac{C\left(l_{0}\right)^{2}}{\delta_{3}}\left\|u_{x}+v+l w\right\|_{2}^{2}+\frac{\delta_{3} c_{p}}{4}\left\|v_{x}\right\|_{2}^{2} \\
& +\frac{C A_{\alpha}}{\delta_{3}}\left(h \diamond\left(u_{x}+v+l w\right)\right)(t), \\
J_{14} \leq & \frac{\delta_{3} c_{p}}{4}\left\|v_{x}\right\|_{2}^{2}+\frac{C \gamma^{2}}{\delta_{3}}\left\|\theta_{x}\right\|_{2}^{2} .
\end{aligned}
$$


We substitute the estimates in (3.22) into (3.21) and choose $\delta_{3}=\frac{k_{2}}{2 c_{p}}$ to obtain the result in (3.20). This completes the proof.

Lemma 3.6 The functional $F_{5}$ defined by

$$
F_{5}(t)=\rho_{3} \int_{0}^{L} v_{t} \int_{0}^{x} \theta(y, t) d y d x
$$

satisfies, for any positive $\epsilon_{5}$ and $\epsilon_{6}$, along the solution of (1.8)-(1.10), the estimate

$$
\begin{aligned}
F_{5}^{\prime}(t) \leq & -\frac{\gamma}{2}\left\|v_{t}\right\|_{2}^{2}+\epsilon_{5}\left\|v_{x}\right\|_{2}^{2}+\epsilon_{6}\left\|u_{x}+v+l w\right\|_{2}^{2}+C\left(1+\frac{1}{\epsilon_{5}}+\frac{1}{\epsilon_{6}}\right)\left\|\theta_{x}\right\|_{2}^{2} \\
& +C A_{\alpha}\left(h \diamond\left(u_{x}+v+l w\right)\right)(t), \quad \forall t \geq 0 .
\end{aligned}
$$

Proof Differentiating $F_{5}$, using $(1.8)_{2}$ and $(1.8)_{4}$, then integration by parts and recalling remark (2.2) and (2.13) give

$$
\begin{aligned}
F_{5}^{\prime}(t)= & \rho_{3} \int_{0}^{L} v_{t} \int_{0}^{x} \theta_{t}(y, t) d y d x+\rho_{3} \int_{0}^{L} v_{t t} \int_{0}^{x} \theta(y, t) d y d x \\
= & -\gamma\left\|v_{t}\right\|_{2}^{2}+\underbrace{\beta \int_{0}^{L} v_{t} \theta_{x} d x}_{J_{15}}-\underbrace{\frac{k_{2} \rho_{3}}{\rho_{2}} \int_{0}^{L} v_{x} \theta d x}_{J_{16}} \\
& -\underbrace{\frac{k_{1} \rho_{3}}{\rho_{2}} \int_{0}^{L}\left(u_{x}+v+l w\right) \int_{0}^{x} \theta(y, t) d y d x}_{J_{17}} \\
& -\underbrace{\frac{\rho_{3}}{\rho_{2}} \int_{0}^{L}\left(g \circ\left(u_{x}+v+l w\right)\right)(x, s, t) \int_{0}^{x} \theta(y, t) d y d x}_{J_{18}} \\
& +\underbrace{\frac{\rho_{3}}{\rho_{2}}\left(\int_{0}^{t} g(s) d s\right) \int_{0}^{L}\left(u_{x}+v+l w\right) \int_{0}^{x} \theta(y, t) d y d x}_{J_{19}}+\frac{\gamma \rho_{3}}{\rho_{2}}\|\theta\|_{2}^{2} .
\end{aligned}
$$


Applying the Cauchy-Schwarz, Young, Poincaré inequalities and Lemmas 2.1-2.2, we estimate $J_{15}-J_{19}$ as follows:

$$
\begin{aligned}
J_{15} & \leq \frac{\gamma}{2}\left\|v_{t}\right\|_{2}^{2}+\frac{\beta^{2}}{2 \gamma}\left\|\theta_{x}\right\|_{2}^{2}, \\
J_{16} & \leq \epsilon_{5}\left\|v_{x}\right\|_{2}^{2}+\frac{c_{p}\left(k_{2} \rho_{3}\right)^{2}}{4 \epsilon_{5} \rho^{2}}\left\|\theta_{x}\right\|_{2}^{2}, \\
J_{17} & \leq \frac{\epsilon_{6}}{2}\left\|u_{x}+v+l w\right\|_{2}^{2}+\frac{\left(k_{1} \rho_{3}\right)^{2}}{2 \epsilon_{6} \rho_{2}^{2}} \int_{0}^{L}\left(\int_{0}^{x} \theta(y, t) d y\right)^{2} d x \\
& \leq \frac{\epsilon_{6}}{2}\left\|u_{x}+v+l w\right\|_{2}^{2}+\frac{c_{p}\left(k_{1} \rho_{3}\right)^{2}}{2 \epsilon_{6} \rho_{2}^{2}}\left\|\theta_{x}\right\|_{2}^{2}, \\
J_{18} & \leq \frac{\rho_{3}}{2 \rho_{2}} \int_{0}^{L}\left(\left(g \circ\left(u_{x}+v+l w\right)\right)(x, s, t)\right)^{2} d x+\frac{\rho_{3}}{2 \rho_{2}} \int_{0}^{L}\left(\int_{0}^{x} \theta(y, t) d y\right)^{2} d x \\
& \leq \frac{\rho_{3} A_{\alpha}}{2 \rho_{2}}\left(h \diamond\left(u_{x}+v+l w\right)\right)(t)+\frac{c_{p} \rho_{3}}{2 \rho_{2}}\left\|\theta_{x}\right\|_{2}^{2}, \\
J_{19} & \leq \frac{\epsilon_{6}}{2}\left\|u_{x}+v+l w\right\|_{2}^{2}+\frac{\rho_{3}^{2}}{2 \epsilon_{6} \rho_{2}^{2}}\left(\int_{0}^{t} g(s) d s\right)^{2} \int_{0}^{L}\left(\int_{0}^{x} \theta(y, t) d y\right)^{2} d x \\
& \leq \frac{\epsilon_{6}}{2}\left\|u_{x}+v+l w\right\|_{2}^{2}+\frac{c_{p}\left(\rho_{3} l_{0}\right)^{2}}{2 \epsilon_{6} \rho_{2}^{2}}\left\|\theta_{x}\right\|_{2}^{2} .
\end{aligned}
$$

By substituting the estimates in (3.25) into (3.24), we get (3.23). This completes the proof.

Lemma 3.7 Let $(u, v, w, \theta)$ be the solution of (1.8)-(1.10), and suppose $k_{1}=k_{3}$. Then the functional $F_{6}$ defined by

$$
\begin{aligned}
F_{6}(t)= & -\rho_{1} \int_{0}^{L} u_{t}\left(w_{x}-l u\right) d x-\rho_{1} \int_{0}^{L} w_{t}\left(u_{x}+v+l w\right) d x \\
& +\frac{\rho_{1}}{k_{3}} \int_{0}^{L} w_{t} \int_{0}^{t} g(t-s)\left(u_{x}+v+l w\right)(x, s) d s d x
\end{aligned}
$$

satisfies the estimate

$$
\begin{aligned}
F_{6}^{\prime}(t) \leq & -\frac{\rho_{1} l}{2}\left\|w_{t}\right\|_{2}^{2}+C\left\|v_{t}\right\|_{2}^{2}+C l\left\|u_{x}+v+l w\right\|_{2}^{2}+\rho_{1} l\left\|u_{t}\right\|_{2}^{2} \\
& -k_{3} l\left\|w_{x}-l u\right\|_{2}^{2}+C\left(1+A_{\alpha}\right)\left(h \diamond\left(u_{x}+v+l w\right)\right)(t), \quad \forall t \geq 0 .
\end{aligned}
$$

Proof Derivation of $F_{6}$, using (1.8), integration by parts, and the fact that $K_{1}=k_{3}$, we get

$$
\begin{aligned}
F_{6}^{\prime}(t)= & -\rho_{1} l\left\|w_{t}\right\|_{2}^{2}-k_{3} l\left\|w_{x}-l u\right\|_{2}^{2}+\rho_{1} l\left\|u_{t}\right\|_{2}^{2}+k_{1} l\left\|u_{x}+v+l w\right\|_{2}^{2} \\
& \underbrace{-\rho_{1} \int_{0}^{L} w_{t} v_{t} d x}_{J_{20}} \underbrace{x-l \int_{0}^{L}\left(u_{x}+v+l w\right) \int_{0}^{t}\left(u_{x}+v+l w\right)(x, s) d s d x}_{J_{21}} \\
& +\underbrace{\frac{g(0) \rho_{1}}{k_{3}} \int_{0}^{L} w_{t}\left(u_{x}+v+l w\right) d x}_{J_{22}}
\end{aligned}
$$




$$
\begin{aligned}
& +\underbrace{k_{1} l \int_{0}^{L}\left(\int_{0}^{t} g(t-s)\left(u_{x}+v+l w\right)(x, s) d s\right)^{2} d x}_{J_{23}} \\
& +\underbrace{\frac{\rho_{1}}{k_{3} \int_{0}^{L} w_{t} \int_{0}^{t} g^{\prime}(t-s)\left(u_{x}+v+l w\right)(x, s) d s d x}}_{J_{24}} \\
& \underbrace{k_{1} l \int_{0}^{L}\left(u_{x}+v+l w\right) \int_{0}^{t} g(t-s)\left(u_{x}+v+l w\right)(x, s) d s d x}_{J_{25}} .
\end{aligned}
$$

Applying the Cauchy-Schwarz, Young inequalities and Lemmas 2.1-2.2, we estimate $J_{20}-$ $J_{25}$ :

$$
\begin{aligned}
J_{20} \leq & \frac{\delta_{4}}{6}\left\|w_{t}\right\|_{2}^{2}+\frac{C}{\delta_{4}}\left\|v_{t}\right\|_{2}^{2} \\
J_{21} \leq & C\left\|u_{x}+v+l w\right\|_{2}^{2}+C A_{\alpha}\left(h \diamond\left(u_{x}+v+l w\right)\right)(t) \\
J_{22} \leq & \frac{\delta_{4}}{6}\left\|w_{t}\right\|_{2}^{2}+\frac{C}{\delta_{4}}\left\|u_{x}+v+l w\right\|_{2}^{2} \\
J_{23} \leq & C\left\|u_{x}+v+l w\right\|_{2}^{2}+C A_{\alpha}\left(h \diamond\left(u_{x}+v+l w\right)\right)(t) \\
J_{24}= & \frac{\rho_{1} \alpha}{k_{3}} \int_{0}^{L} w_{t} \int_{0}^{t} g(t-s)\left(u_{x}+v+l w\right)(x, s) d s d x \\
& -\frac{\rho_{1}}{k_{3}} \int_{0}^{L} w_{t} \int_{0}^{t} h(t-s)\left(u_{x}+v+l w\right)(x, s) d s d x \\
\leq & \frac{2 \delta_{4}}{3}\left\|w_{t}\right\|_{2}^{2}+\frac{C}{\delta_{4}}\left\|u_{x}+v+l w\right\|_{2}^{2}+C \frac{\left(A_{\alpha}+1\right)}{\delta_{4}}\left(h \diamond\left(u_{x}+v+l w\right)\right)(t)
\end{aligned}
$$

$J_{25} \leq C\left\|u_{x}+v+l w\right\|_{2}^{2}+C A_{\alpha}\left(h \diamond\left(u_{x}+v+l w\right)\right)(t)$.

Substituting the estimates in (3.28) into (3.27) and selecting $\delta_{4}=\frac{\rho_{1} l}{2}$, we obtain (3.26). This completes the proof.

Lemma 3.8 The functional $F_{7}$ defined by

$$
F_{7}(t)=\int_{0}^{L} \int_{0}^{t} J(t-s)\left(u_{x}+v+l w\right)^{2}(x, s) d s d x, \quad \text { where } J(t)=\int_{t}^{+\infty} g(s) d s
$$

satisfies, along the solution of (1.8)-(1.10), the estimate

$$
F_{7}^{\prime}(t) \leq-\frac{1}{2}\left(g \diamond\left(u_{x}+v+l w\right)\right)(t)+3\left(1-l_{0}\right)\left\|u_{x}+v+l w\right\|_{2}^{2}, \quad \forall t \geq 0 .
$$

Proof By differentiating $F_{7}$ and recalling that

$$
J^{\prime}(t)=-g(t) \text { and } \quad J(t)=J(0)-\int_{0}^{t} g(s) d s
$$


we have

$$
\begin{aligned}
F_{7}^{\prime}(t)= & \int_{0}^{L} \int_{0}^{t} J^{\prime}(t-s)\left(u_{x}+v+l w\right)^{2}(x, s) d s d x+J(0)\left\|u_{x}+v+l w\right\|_{2}^{2} \\
= & -\int_{0}^{L} \int_{0}^{t} g(t-s)\left(u_{x}+v+l w\right)^{2}(x, s) d s d x+J(t)\left\|u_{x}+v+l w\right\|_{2}^{2} \\
& +\int_{0}^{t} g(s) d s\left\|u_{x}+v+l w\right\|_{2}^{2} \\
= & -\int_{0}^{L} \int_{0}^{t} g(t-s)\left(\left(u_{x}+v+l w\right)(x, t)-\left(u_{x}+v+l w\right)(x, s)\right)^{2} d s d x \\
& +J(t)\left\|u_{x}+v+l w\right\|_{2}^{2} \\
& -2 \int_{0}^{L}\left(u_{x}+v+l w\right) \int_{0}^{t} g(t-s)\left(\left(u_{x}+v+l w\right)-\left(u_{x}+v+l w\right)\right) d s d x \\
\leq & -\left(g \diamond\left(u_{x}+v+l w\right)\right)(t)+J(t)\left\|u_{x}+v+l w\right\|_{2}^{2} \\
& +2\left(1-l_{0}\right)\left\|u_{x}+v+l w\right\|_{2}^{2}+\frac{\left(\int_{0}^{t} g(s) d s\right)}{2\left(1-l_{0}\right)}\left(g \diamond\left(u_{x}+v+l w\right)\right)(t) \\
\leq & -\frac{1}{2}\left(g \diamond\left(u_{x}+v+l w\right)\right)(t)+2\left(1-l_{0}\right)\left\|u_{x}+v+l w\right\|_{2}^{2}+J(t)\left\|u_{x}+v+l w\right\|_{2}^{2} .
\end{aligned}
$$

Recalling that $J^{\prime}(t)=-g(t) \leq 0$, then $J$ is nonincreasing, hence

$$
J(t) \leq J(0)=\left(1-l_{0}\right) .
$$

The result follows.

Lemma 3.9 Let $(u, v, w, \theta)$ be the solution of (1.8)-(1.10), and assume $k_{1}=k_{3}$. Then, for $l$ small enough and suitable choices of $N, N_{j}, j=1, \ldots, 6$, the Lyapunov functional L defined by

$$
L(t)=N E(t)+\sum_{j=1}^{6} N_{j} F_{j}(t)
$$

satisfies the estimates

$$
\alpha_{1} E(t) \leq L(t) \leq \alpha_{2} E(t)
$$

and

$$
\begin{aligned}
L^{\prime}(t) \leq & -\lambda\left(\left\|u_{t}\right\|_{2}^{2}+\left\|v_{t}\right\|_{2}^{2}+\left\|w_{t}\right\|_{2}^{2}+\left\|u_{x}+v+l w\right\|_{2}^{2}+\left\|v_{x}\right\|_{2}^{2}\right) \\
& -\lambda\left(\left\|w_{x}-l u\right\|_{2}^{2}+\left\|\theta_{x}\right\|_{2}^{2}\right)+\frac{1}{4}\left(g \diamond\left(u_{x}+v+l w\right)\right)(t), \quad \forall t \geq t_{0},
\end{aligned}
$$

for some $\lambda>0$ and $\alpha_{1}, \alpha_{2}>0$.

Proof Applying the Cauchy-Schwarz, Young, and Poincaré inequalities, we get (3.32) from routine calculations. Recalling that $h=\alpha g-g^{\prime}$, it follows from Lemmas 3.1-3.7 that, 
for any $t \geq t_{0}$,

$$
\begin{aligned}
L^{\prime}(t) \leq & -\left[\frac{N_{3} \rho_{1} g_{0}}{2}-N_{1} \epsilon_{0}-N_{2}\left(\rho_{1}+\frac{\rho_{1}^{2}}{4 \epsilon_{1}}+\frac{\left(\rho_{1} l\right)^{2}}{4 \epsilon_{2}}\right)-\frac{N_{6} k_{3} \rho_{1} l}{k_{1}}\right]\left\|u_{t}\right\|_{2}^{2} \\
& -\left[\frac{\gamma N_{5}}{2}-N_{2} \epsilon_{1}-N_{3} C-N_{4} \rho_{2}-N_{6} C\right]\left\|v_{t}\right\|_{2}^{2} \\
& -\left[\frac{N_{6} \rho_{1} l}{2}-N_{1}\left(\rho_{1}+\frac{\left(\rho_{1} l\right)^{2}}{4 \epsilon_{0}}\right)-N_{2} \epsilon_{2}-N_{3} C l^{2}\right]\left\|w_{t}\right\|_{2}^{2} \\
& -\left[\frac{N_{2} l_{0}}{2}-N_{1} C l-N_{3} \epsilon_{3}-N_{4} C-N_{5} \epsilon_{6}-N_{6} C\right]\left\|u_{x}+v+l w\right\|_{2}^{2} \\
& -\left[\frac{N_{4} k_{2}}{2}-N_{5} \epsilon_{5}\right]\left\|v_{x}\right\|_{2}^{2}-\left[\frac{N_{1} k_{3}}{2}+N_{6} k_{3} l-N_{2} C l-N_{3} \epsilon_{4}\right]\left\|w_{x}-l u\right\|_{2}^{2} \\
& -\left[N \beta-N_{4} C-N_{5} C\left(1+\frac{1}{\epsilon_{5}}+\frac{1}{\epsilon_{6}}\right)\right]\left\|\theta_{x}\right\|_{2}^{2} \\
& +\frac{N \alpha}{2}\left(g \diamond\left(u_{x}+v+l w\right)\right)(t) \\
& -\left[\frac{N}{2}-C A_{\alpha}\left(N_{1}+N_{2}+N_{3}\left(1+\frac{1}{\epsilon_{3}}+\frac{1}{\epsilon_{4}}\right)+N_{4}+N_{5}+N_{6}\right)-N_{6} C\right] \\
& \times\left(h \diamond\left(u_{x}+v+l w\right)\right)(t) .
\end{aligned}
$$

\section{Choosing}

$$
\begin{aligned}
& N_{4}=N_{1}=1, \quad \epsilon_{0}=\frac{N_{3} \rho_{1} g_{0}}{4 N_{1}}, \quad \epsilon_{2}=\frac{N_{6} \rho_{1} l}{4 N_{2}}, \quad \epsilon_{3}=\frac{N_{2} l_{0}}{8 N_{3}}, \\
& \epsilon_{4}=\frac{N_{1} k_{3}}{4 N_{3}}, \quad \epsilon_{5}=\frac{N_{4} k_{2}}{4 N_{5}}, \quad \epsilon_{6}=\frac{N_{2} l_{0}}{8 N_{5}},
\end{aligned}
$$

inequality (3.34) yields

$$
\begin{aligned}
L^{\prime}(t) \leq & -\left[\frac{N_{3} \rho_{1} g_{0}}{4}-N_{2}\left(\rho_{1}+\frac{\rho_{1}^{2}}{4 \epsilon_{1}}+\frac{\left(\rho_{1} l\right)^{2}}{4 \epsilon_{2}}\right)-\frac{N_{6} k_{3} \rho_{1} l}{k_{1}}\right]\left\|u_{t}\right\|_{2}^{2} \\
& -\left[\frac{\gamma N_{5}}{2}-N_{2} \epsilon_{1}-N_{3} C-N_{4} \rho_{2}-N_{6} C\right]\left\|v_{t}\right\|_{2}^{2} \\
& -\left[\frac{N_{6} \rho_{1} l}{4}-\left(\rho_{1}+\frac{\left(\rho_{1} l\right)^{2}}{4 \epsilon_{0}}\right)-N_{3} C l^{2}\right]\left\|w_{t}\right\|_{2}^{2} \\
& -\left[\frac{N_{2} k_{1} l_{0}}{4}-C l-C-N_{6} C\right]\left\|u_{x}+v+l w\right\|_{2}^{2} \\
& -\frac{k_{2}}{4}\left\|v_{x}\right\|_{2}^{2}-\left[\frac{k_{3}}{4}+\frac{N_{6} k_{3}^{2} l}{k_{1}}-N_{2} C l\right]\left\|w_{x}-l u\right\|_{2}^{2} \\
& -\left[N \beta-N_{4} C-N_{5} C\left(1+\frac{1}{\epsilon_{5}}+\frac{1}{\epsilon_{6}}\right)\right]\left\|\theta_{x}\right\|_{2}^{2}+\frac{N k_{1} \alpha}{2}\left(g \diamond\left(u_{x}+v+l w\right)\right)(t) \\
& -\left[\frac{N k_{1}}{2}-C A_{\alpha}\left(2+N_{2}+N_{3}\left(1+\frac{1}{\epsilon_{3}}+\frac{1}{\epsilon_{4}}\right)+N_{5}+N_{6}\right)-N_{6} C\right] \\
& \times\left(h \diamond\left(u_{x}+v+l w\right)\right)(t) .
\end{aligned}
$$


Now, we select the constants in (3.36) carefully. First, we choose $N_{6}$ so large that

$$
\mu_{1}:=\frac{N_{6} \rho_{1} l}{4}-\left(\rho_{1}+\frac{\left(\rho_{1} l\right)^{2}}{4 \epsilon_{0}}\right)>0,
$$

then we select $N_{2}$ large enough such that

$$
\mu_{2}:=\frac{N_{2} l_{0}}{4}-C-N_{6} C>0
$$

Next, we select $N_{3}$ large enough such that

$$
\mu_{3}:=\frac{N_{3} \rho_{1} g_{0}}{4}-N_{2}\left(\rho_{1}+\frac{\rho_{1}^{2}}{4 \epsilon_{1}}+\frac{\left(\rho_{1} l\right)^{2}}{4 \epsilon_{2}}\right)>0 .
$$

Now, we choose $N_{5}$ large enough so that

$$
\mu_{4}:=\frac{\gamma N_{5}}{2}-N_{3} C-\rho_{2}-N_{6} C>0
$$

We then select $l$ and $\epsilon_{1}$ small enough so that

$$
\begin{aligned}
& \mu_{1}-N_{3} C l^{2}>0, \quad \mu_{2}-C l>0, \quad \mu_{3}-\frac{N_{6} k_{3} \rho_{1} l}{k_{1}}>0, \\
& \frac{k_{3}}{4}-N_{2} C l>0, \quad \text { and } \quad \mu_{4}-N_{2} \epsilon_{1}>0 .
\end{aligned}
$$

Recalling that $h=\alpha g-g^{\prime}$, we have $\frac{\alpha g^{2}(s)}{h(s)}=\frac{\alpha g^{2}(s)}{\alpha g(s)-g^{\prime}(s)}<g(s)$; thus application of dominated convergence theorem gives

$$
\alpha A_{\alpha}=\int_{0}^{+\infty} \frac{\alpha g^{2}(s)}{\alpha g(s)-g^{\prime}(s)} d s \rightarrow 0 \quad \text { as } \alpha \rightarrow 0 .
$$

Therefore, we can find $0<\alpha_{0}<1$ so that if $\alpha<\alpha_{0}<1$, then

$$
\alpha A_{\alpha}<\frac{1}{2 C\left(2+N_{2}+N_{3}\left(1+\frac{1}{\epsilon_{3}}+\frac{1}{\epsilon_{4}}\right)+N_{5}+N_{6}\right)} .
$$

Finally, we choose $N$ so large and take $\alpha=\frac{1}{2 N}$ such that (3.32) remains true and

$$
\mu_{5}:=N \beta-N_{4} C-N_{5} C\left(1+\frac{1}{\epsilon_{5}}+\frac{1}{\epsilon_{6}}\right)>0
$$

as well as

$$
\mu_{6}:=\frac{N k_{1}}{2}-C A_{\alpha}\left(2+N_{2}+N_{3}\left(1+\frac{1}{\epsilon_{3}}+\frac{1}{\epsilon_{4}}\right)+N_{5}+N_{6}\right)-N_{6} C>0 .
$$

A combination of (3.35)-(3.44) yields (3.33). 


\section{Main decay result}

Now, we state the main stability result of this paper.

Theorem 4.1 Let $(u, v, w, \theta)$ be the solution of (1.8)-(1.10). Suppose that $k_{3}=k_{1}$ and assumptions $\left(A_{1}\right)$ and $\left(A_{2}\right)$ hold. Then, for $l$ small enough, the solution energy functional (3.2) satisfies

$$
E(t) \leq M_{2} H_{1}^{-1}\left(M_{1} \int_{t_{0}}^{t} \xi(s) d s\right), \quad \text { where } H_{1}(t)=\int_{t}^{r} \frac{1}{s H^{\prime}(s)} d s
$$

for some positive constants $M_{1}$ and $M_{2}$, and $H_{1}$ is a strictly convex function that is nonincreasing on $(0, r]$, where $r=g\left(t_{0}\right)>0$ and $\lim _{t \rightarrow 0} H_{1}(t)=+\infty$.

Remark 4.1 The decay result in (4.1) is optimal in the sense that it agrees with the decay rate of $g$, see [27], Remark 2.3.

Corollary 4.1 Suppose that $k_{3}=k_{1}$ and assumptions $\left(A_{1}\right)$ and $\left(A_{2}\right)$ hold. Assume that the function $\mathrm{H}$ in assumption $\left(A_{2}\right)$ is defined by

$$
H(s)=s^{q}, \quad 1 \leq q<2 .
$$

Then the solution energy (3.2) satisfies

$$
E(t) \leq \begin{cases}M \exp \left(-\bar{M} \int_{0}^{t} \xi(s) d s\right) & \text { for } q=1, \\ E(t) \leq \frac{M}{\left(1+\int_{t_{0}}^{t} \xi(s) d s\right)^{\frac{1}{q-1}}} & \text { for } 1<q<2\end{cases}
$$

for some positive constants $M$ and $\bar{M}$.

\subsection{Examples}

(1) Let $g_{1}(t)=a_{0} e^{-a_{1} t}, t \geq 0, a_{0}, a_{1}>0$ be constants and $a_{0}$ be chosen in a way that $\left(A_{1}\right)$ holds. Then

$$
g_{1}^{\prime}(t)=-a_{0} a_{1} e^{-a_{1} t}=-a_{1} H\left(g_{1}(t)\right), \quad \text { where } H(s)=s .
$$

In this case, the solution energy (3.2) 1 satisfies

$$
E(t) \leq M e^{-\lambda t}, \quad \forall t \geq 0,
$$

for some positive constants $M, \lambda$.

(2) Let $g_{2}(t)=a_{0} e^{-(1+t)^{a_{1}}}, t \geq 0, a_{0}>0,0<a_{1}<1$ be constants and $a_{0}$ be chosen in a way that $\left(A_{1}\right)$ holds. Then

$$
g_{2}^{\prime}(t)=-a_{0} a_{1}(1+t)^{a_{1}-1} e^{-(1+t)^{a_{1}}}=-\xi(t) H\left(g_{2}(t)\right),
$$

where

$$
\xi(t)=a_{1}(1+t)^{a_{1}-1}, \quad H(s)=s .
$$


Here, the solution energy $(3.2)_{1}$ satisfies

$$
E(t) \leq M e^{-\lambda(1+t)^{a_{1}}}, \quad \forall t \geq 0,
$$

for some positive constants $M, \lambda$.

(3) Let $g_{3}(t)=\frac{a_{0}}{(1+t)^{a_{1}}}, t \geq 0, a_{0}>0, a_{1}>1$ be constants and $a_{0}$ be chosen in a way that $\left(A_{1}\right)$ holds. Then

$$
g_{3}^{\prime}(t)=\frac{-a_{0} a_{1}}{(1+t)^{a_{1}+1}}=-a_{1} H\left(g_{3}(t)\right), \quad \text { where } H(s)=s^{q}, q=\frac{a_{1}+1}{a_{1}}
$$

with $q$ satisfying $1<q<2$. Thus, the solution energy (3.2)

$$
E(t) \leq \frac{M}{(1+t)^{a_{1}}}
$$

for some positive constant $M$.

\subsection{Proof of Theorem 4.1}

Proof Using (2.6) and (3.2), we obtain

$$
\begin{aligned}
& \int_{0}^{t_{0}} g(s)\left\|\left(u_{x}+v+l w\right)(t)-\left(u_{x}+v+l w\right)(t-s)\right\|_{2}^{2} d s \\
& \quad \leq-\frac{g(0)}{b_{1}} \int_{0}^{t_{0}} g^{\prime}(s)\left\|\left(u_{x}+v+l w\right)(t)-\left(u_{x}+v+l w\right)(t-s)\right\|_{2}^{2} d s \\
& \quad \leq-C E^{\prime}(t), \quad \forall t \in\left[0, t_{0}\right] .
\end{aligned}
$$

Thus, from (2.11), (3.33), and (4.4), we have

$$
\begin{aligned}
L^{\prime}(t) \leq & -\lambda E(t)+\frac{1}{4}\left(g \diamond\left(u_{x}+v+l w\right)(t)\right. \\
= & -\lambda E(t)+\frac{1}{4} \int_{0}^{t_{0}} g(s)\left\|\left(u_{x}+v+l w\right)(t)-\left(u_{x}+v+l w\right)(t-s)\right\|_{2}^{2} d s \\
& +\frac{1}{4} \int_{t_{0}}^{t} g(s)\left\|\left(u_{x}+v+l w\right)(t)-\left(u_{x}+v+l w\right)(t-s)\right\|_{2}^{2} d s \\
\leq & -\lambda E(t)-C E^{\prime}(t) \\
& +\frac{1}{4} \int_{t_{0}}^{t} g(s)\left\|\left(u_{x}+v+l w\right)(t)-\left(u_{x}+v+l w\right)(t-s)\right\|_{2}^{2} d s .
\end{aligned}
$$

Therefore, we obtain

$$
L_{1}^{\prime}(t) \leq-\lambda E(t)+\frac{1}{4} \int_{t_{0}}^{t} g(s)\left\|\left(u_{x}+v+l w\right)(t)-\left(u_{x}+v+l w\right)(t-s)\right\|_{2}^{2} d s, \quad \forall t \geq t_{0}
$$

where $L_{1}(t)=L(t)+C E(t) \sim E(t)$ because of (3.32). To complete the proof, we discuss two cases. 
Case1: $\mathbf{H}(\mathbf{t})$ is linear. By multiplying (4.5) by $\xi(t)$, keeping in mind (3.2) and $\left(A_{2}\right)$, we get

$$
\begin{aligned}
\xi(t) L_{1}^{\prime}(t) \leq & -\lambda \xi(t) E(t) \\
& +\frac{1}{4} \xi(t) \int_{t_{0}}^{t} g(s)\left\|\left(u_{x}+v+l w\right)(t)-\left(u_{x}+v+l w\right)(t-s)\right\|_{2}^{2} d s \\
\leq & -\lambda \xi(t) E(t) \\
& +\frac{1}{2} \int_{t_{0}}^{t} \xi(s) g(s)\left\|\left(u_{x}+v+l w\right)(t)-\left(u_{x}+v+l w\right)(t-s)\right\|_{2}^{2} d s \\
\leq & -\lambda \xi(t) E(t) \\
& -\frac{1}{2} \int_{t_{0}}^{t} g^{\prime}(s)\left\|\left(u_{x}+v+l w\right)(t)-\left(u_{x}+v+l w\right)(t-s)\right\|_{2}^{2} d s \\
\leq & -\lambda \xi(t) E(t)-C E^{\prime}(t) .
\end{aligned}
$$

Recalling that $\xi$ is nonincreasing, we arrive at

$$
\left(\xi L_{1}+C E\right)^{\prime}(t) \leq-\lambda \xi(t) E(t), \quad \forall t \geq t_{0}
$$

and since $L_{1} \sim E$, we get

$$
\xi L_{1}+C E \sim E
$$

Thus, for $L_{2}(t)=\xi(t) L_{1}(t)+C E(t)$, we obtain

$$
L_{2}^{\prime}(t) \leq-\lambda \xi(t) E(t) \leq-M \xi(t) L_{2}(t), \quad \forall t \geq t_{0}
$$

where $M$ is a positive constant. Integrating (4.9) over $\left(t_{0}, t\right)$ and recalling (4.8) give

$$
E(t) \leq M \exp \left(-M^{\prime} \int_{t_{0}}^{t} \xi(s) d s\right)=M_{2} H_{1}^{-1}\left(M_{1} \int_{t_{0}}^{t} \xi(s) d s\right) .
$$

Case 2: $\mathbf{H}(\mathbf{t})$ is nonlinear. In this case, let us define the functional $\mathcal{L}(t)=L(t)+F_{7}(t)$. Then we obtain, for some positive constant $d>0$,

$$
\mathcal{L}^{\prime}(t) \leq-d E(t), \quad \forall t \geq t_{0}
$$

The last inequality implies

$$
d \int_{t_{0}}^{t} E(s) d s \leq \mathcal{L}\left(t_{0}\right)-\mathcal{L}(t) \leq \mathcal{L}\left(t_{0}\right)
$$

Thus, we obtain

$$
\int_{0}^{+\infty} E(s) d s<\infty
$$


Now, we define the functional $\Phi$

$$
\Phi(t):=p \int_{t_{0}}^{t}\left\|\left(u_{x}+v+l w\right)(t)-\left(u_{x}+v+l w\right)(t-s)\right\|_{2}^{2} d s
$$

Using (4.11), we can choose $p$ such that $0<p<1$ and

$$
\Phi(t)<1, \quad \forall t \geq t_{0}
$$

Note that without loss of generality we assume $\Phi(t)>0, \forall t \geq t_{0}$; otherwise, we immediately get from (4.5) an exponential stability result. Also, we define the functional $\Psi$ by

$$
\Psi(t)=-\int_{t_{0}}^{t} g^{\prime}(s)\left\|\left(u_{x}+v+l w\right)(t)-\left(u_{x}+v+l w\right)(t-s)\right\|_{2}^{2} d s .
$$

From (3.1), we get $\Psi(t) \leq-C E^{\prime}(t), \forall t \geq t_{0}$. Using assumption $\left(A_{2}\right)$, we observe that $H$ is strictly convex on $(0, r], r=g\left(t_{0}\right)$ and $H(0)=H^{\prime}(0)=0$. Therefore, we get

$$
H(\mu s) \leq \mu H(s), \quad 0 \leq \mu \leq 1, s \in(0, r]
$$

Thus, it follows from assumption $\left(A_{2}\right),(4.12)$, and Lemma 2.3 that

$$
\begin{aligned}
\Psi(t) & =\frac{1}{p \Phi(t)} \int_{t_{0}}^{t} \Phi(t)\left(-g^{\prime}(s)\right) p\left\|\left(u_{x}+v+l w\right)(t)-\left(u_{x}+v+l w\right)(t-s)\right\|_{2}^{2} d s \\
& \geq \frac{1}{p \Phi(t)} \int_{t_{0}}^{t} \Phi(t) \xi(s) H(g(s)) p\left\|\left(u_{x}+v+l w\right)(t)-\left(u_{x}+v+l w\right)(t-s)\right\|_{2}^{2} d s \\
& \geq \frac{\xi(t)}{p \Phi(t)} \int_{t_{0}}^{t} H(\Phi(t) g(s)) p\left\|\left(u_{x}+v+l w\right)(t)-\left(u_{x}+v+l w\right)(t-s)\right\|_{2}^{2} d s \\
& \geq \frac{\xi(t)}{p} H\left(\int_{t_{0}}^{t} g(s) \alpha\left\|\left(u_{x}+v+l w\right)(t)-\left(u_{x}+v+l w\right)(t-s)\right\|_{2}^{2} d s\right) \\
& =\frac{\xi(t)}{p} H\left(p \int_{t_{0}}^{t} g(s)\left\|\left(u_{x}+v+l w\right)(t)-\left(u_{x}+v+l w\right)(t-s)\right\|_{2}^{2} d s\right) \\
& =\frac{\xi(t)}{p} \bar{H}\left(p \int_{t_{0}}^{t} g(s)\left\|\left(u_{x}+v+l w\right)(t)-\left(u_{x}+v+l w\right)(t-s)\right\|_{2}^{2} d s\right),
\end{aligned}
$$

where $\bar{H}$ is the extension of $H$ on $(0,+\infty)$, see (2.3). Using (4.14), we have $\forall t \geq t_{0}$

$$
\int_{t_{0}}^{t} g(s)\left\|\left(u_{x}+v+l w\right)(t)-\left(u_{x}+v+l w\right)(t-s)\right\|_{2}^{2} d s \leq \frac{1}{p} \bar{H}^{-1}\left(\frac{p \Psi(t)}{\xi(t)}\right) .
$$

It follows from (4.5) that

$$
L_{1}^{\prime}(t) \leq-\lambda E(t)+C \bar{H}^{-1}\left(\frac{p \Psi(t)}{\xi(t)}\right), \quad \forall t \geq t_{0} .
$$

Let $r_{0}<r$ be specified later and define the functional $U_{2}$ by

$$
U_{2}(t):=\bar{H}^{\prime}\left(r_{0} \frac{E(t)}{E(0)}\right) L_{1}(t)+E(t)
$$


Then $U_{2} \sim E$ since $L_{1} \sim E$. From (4.15) and using the fact that

$$
E^{\prime}(t) \leq 0, \quad \bar{H}^{\prime}(t)>0, \quad \bar{H}^{\prime \prime}(t)>0,
$$

we arrive at

$$
\begin{aligned}
U_{2}^{\prime}(t) & =r_{0} \frac{E^{\prime}(t)}{E(0)} \bar{H}^{\prime \prime}\left(r_{0} \frac{E(t)}{E(0)}\right) L_{1}(t)+\bar{H}^{\prime}\left(r_{0} \frac{E(t)}{E(0)}\right) L_{1}^{\prime}(t)+E^{\prime}(t) \\
& \leq-\lambda E(t) \bar{H}^{\prime}\left(r_{0} \frac{E(t)}{E(0)}\right)+C \bar{H}^{\prime}\left(r_{0} \frac{E(t)}{E(0)}\right) \bar{H}^{-1}\left(p \frac{\Psi(t)}{\xi(t)}\right)+E^{\prime}(t) .
\end{aligned}
$$

Next, we consider the convex conjugate $\bar{H}^{*}$ of $\bar{H}$ in the sense of Young (see [28] pp. 61-64) defined by

$$
\bar{H}^{*}(s)=s\left(\bar{H}^{\prime}\right)^{-1}(s)-\bar{H}\left[\left(\bar{H}^{\prime}\right)\right],
$$

where $\bar{H}^{*}$ satisfies the generalized Young inequality

$$
X_{1} X_{2} \leq \bar{H}^{*}\left(X_{1}\right)+\bar{H}\left(X_{2}\right) .
$$

We set

$$
X_{1}=\bar{H}^{\prime}\left(r_{0} \frac{E(t)}{E(0)}\right) \quad \text { and } \quad X_{2}=\bar{H}^{-1}\left(p \frac{\Psi(t)}{\xi(t)}\right) .
$$

Then using Lemma 3.1 and (4.16)-(4.18), we obtain for all $t \geq t_{0}$

$$
\begin{aligned}
U_{2}^{\prime}(t) & \leq-\lambda E(t) \bar{H}^{\prime}\left(r_{0} \frac{E(t)}{E(0)}\right)+C \bar{H}^{*}\left(\bar{H}^{\prime}\left(r_{0} \frac{E(t)}{E(0)}\right)\right)+C p \frac{\Psi(t)}{\xi(t)}+E^{\prime}(t) \\
& \leq-\lambda E(t) \bar{H}^{\prime}\left(r_{0} \frac{E(t)}{E(0)}\right)+C r_{0} \frac{E(t)}{E(0)} \bar{H}^{\prime}\left(r_{0} \frac{E(t)}{E(0)}\right)+C p \frac{\Psi(t)}{\xi(t)}+E^{\prime}(t) .
\end{aligned}
$$

Now, we multiply (4.19) by $\xi(t)$ and observe that $r_{0} \frac{E(t)}{E(0)}<r$ and

$$
\bar{H}^{\prime}\left(r_{0} \frac{E(t)}{E(0)}\right)=H^{\prime}\left(r_{0} \frac{E(t)}{E(0)}\right) .
$$

Thus we get for all $t \geq t_{0}$

$$
\begin{aligned}
\xi(t) U_{2}^{\prime}(t) \leq & -\lambda \xi(t) E(t) H^{\prime}\left(r_{0} \frac{E(t)}{E(0)}\right)+C r_{0} \frac{E(t)}{E(0)} \xi(t) H^{\prime}\left(r_{0} \frac{E(t)}{E(0)}\right) \\
& +C p \Psi(t)+\xi(t) E^{\prime}(t) \\
\leq & -\lambda \xi(t) E(t) H^{\prime}\left(r_{0} \frac{E(t)}{E(0)}\right)+C r_{0} \frac{E(t)}{E(0)} \xi(t) H^{\prime}\left(r_{0} \frac{E(t)}{E(0)}\right) \\
& -C E^{\prime}(t) .
\end{aligned}
$$

Let $U_{3}(t)=\xi(t) U_{2}(t)+C E(t)$, then $U_{3}$ satisfies

$$
m_{0} U_{3}(t) \leq E(t) \leq m_{1} U_{3}(t)
$$


for some constants $m_{0}, m_{1}>0$ since $U_{2} \sim E$. From (4.20), we have

$$
U_{3}^{\prime}(t) \leq-\left(\lambda E(0)-C r_{0}\right) \xi(t) \frac{E(t)}{E(0)} \xi(t) H^{\prime}\left(r_{0} \frac{E(t)}{E(0)}\right), \quad \forall t \geq t_{0} .
$$

By choosing $r_{0}<r$ small enough such that $\lambda E(0)-C r_{0}>0$, we obtain for some constant $M>0$

$$
U_{3}^{\prime}(t) \leq-M \xi(t) \frac{E(t)}{E(0)} \xi(t) H^{\prime}\left(r_{0} \frac{E(t)}{E(0)}\right)=-M \xi(t) H_{2}\left(\frac{E(t)}{E(0)}\right), \quad \forall t \geq t_{0}
$$

where

$$
H_{2}(s)=s H^{\prime}\left(r_{0} s\right) .
$$

We observe that

$$
H_{2}^{\prime}(s)=H^{\prime}\left(r_{0} s\right)+r_{0} s H^{\prime \prime}\left(r_{0} s\right) .
$$

Hence, the strict convexity of $H$ on $(0, r]$ implies $H_{2}(s)>0, H_{2}^{\prime}(s)>0$ on $(0, r]$.

Let

$$
W(t)=m_{0} \frac{U_{3}(t)}{E(0)} .
$$

Then, recalling (4.21) and (4.22), we have

$$
W(t) \sim E(t)
$$

and

$$
W^{\prime}(t)=m_{0} \frac{U_{3}^{\prime}(t)}{E(0)} \leq-M_{1} \xi(t) H_{2}(W(t)), \quad \forall t \geq t_{0}
$$

Integration of (4.24) over $\left(t_{0}, t\right)$ gives

$$
M_{1} \int_{t_{0}}^{t} \xi(s) d s \leq-\int_{t_{0}}^{t} \frac{W^{\prime}(s)}{H_{2}(W(s))} d s=\frac{1}{r_{0}} \int_{r_{0} W(t)}^{r_{0} W\left(t_{0}\right)} \frac{1}{s H^{\prime}(s)} d s
$$

which yields

$$
W(t) \leq \frac{1}{r_{0}} H_{1}^{-1}\left(M_{1} \int_{t_{0}}^{t} \xi(s) d s\right), \quad \text { where } H_{1}(t)=\int_{t}^{r} \frac{1}{s H^{\prime}(s)} d s .
$$

Using the assumption on $H$ from $\left(A_{2}\right)$, we see that $H_{1}$ is a strictly decreasing function on $(0, r]$ and

$$
\lim _{t \rightarrow 0} H_{1}(t)=+\infty
$$


Hence, using (4.23) and (4.25), we obtain decay estimate

$$
E(t) \leq M_{2} H_{1}^{-1}\left(M_{1} \int_{t_{0}}^{t} \xi(s) d s\right) .
$$

\section{This completes the proof.}

\section{Acknowledgements}

The authors would like to appreciate the remarks of the anonymous reviewer which have greatly improved the manuscript. The continuous support of University of Hafr Al Batin is appreciated.

\section{Funding}

This work is sponsored by University of Hafr Al Batin, Deanship of Research, Saudi Arabia under project number $G-106-2020$

\section{Availability of data and materials}

Not applicable.

\section{Competing interests}

The authors declare that they have no competing interests.

\section{Authors' contributions}

All authors contributed equally and approved the present status of the manuscript.

\section{Publisher's Note}

Springer Nature remains neutral with regard to jurisdictional claims in published maps and institutional affiliations.

Received: 12 September 2020 Accepted: 12 July 2021 Published online: 04 August 2021

\section{References}

1. Alves, M.O., Fatori, L.H., Silva, M.A.J., Monteriro, R.N.: Stability and optimality of decay rate for a weakly dissipative Bresse system. Math. Methods Appl. Sci. 38, 898-908 (2015)

2. Alves, M.S., Vera, O., Muñoz-Rivera, J.E., Rambaud, A.: Exponential stability to the Bresse system with boundary dissipation conditions (2015) arXiv:1506.01657

3. DellÓro, F: Asymptotic stability of thermoelastic systems of Bresse type. J. Differ. Equ. 258, 3902-3927 (2015)

4. Fatori, L.H., Monteiro, R.N.: The optimal decay rate for a weak dissipative Bresse system. Appl. Math. Lett. 25, 600-604 (2012)

5. Guesmia, A., Kafini, M.: Bresse system with infinite memories. Math. Methods Appl. Sci. 38, 2389-2402 (2015)

6. Ma, T.F., Monteiro, R.N.: Singular limit and long-time dynamics of Bresse systems. SIAM J. Math. Anal. 49, 2468-2495 (2017)

7. Soriano, J.A., Muñoz Rivera, J.E., Fatori, L.H.: Bresse system with indefinite damping. J. Math. Anal. Appl. 387, 284-290 (2012)

8. Soufyane, A., Said-Houari, B.: The effect of the wave speeds and the frictional damping terms on the decay rate of the Bresse system. Evol. Equ. Control Theory 3, 713-738 (2014)

9. Wehbe, A., Youssef, W.: Exponential and polynomial stability of an elastic Bresse system with two locally distributed feedbacks. J. Math. Phys. 51, 1-17 (2010)

10. Bresse, A.J.A.C.: Cours de Méchanique Appliquée. Gauthier-Villars, Paris (1866)

11. Liu, B.Z., Rao, B.: Energy decay rate of the thermoelastic Bresse system. Z. Angew. Math. Phys. 60(1), 54-69 (2009)

12. Fatori, C.L.H., Mu noz Rivera, J.E.: Rates of decay to weak thermoelastic Bresse system. IMA J. Appl. Math. 75(6), 881-904 (2010)

13. Conti, M., Pata, V.: General decay properties of abstract linear viscoelasticity. Z. Angew. Math. Phys. 71, 6 (2020)

14. Messaoudi, S.A., Hassan, J.H.: New general decay result in finite memory of a Bresse system. Commun. Pure Appl. Anal. 18(4), 1637-1662 (2019)

15. Lagnese, J.E., Leugering, G., Schmidt, E.J.P.G.: Modelling of dynamic networks of thin thermoelastic beams. Math. Methods Appl. Sci. 16(5), 327-358 (1993)

16. Lagnese, J.E., Leugering, G., Schmidt, E.J.P.G.: Modeling, Analysis and Control of Dynamic Elastic Multi-Link Structures. Systems and Control: Foundations and Applications. Birkhäuser, Boston (1994)

17. Timoshenko, S.P.: On the correction for shear of the differential equation for transverse vibrations of prismatic bars. Philos. Mag. Ser. 6 41(245), 744-746 (1921)

18. Timoshenko, S.P.: Vibration Problems in Engineering. Van Nostrand, New York (1955)

19. Drozdov, A.D., Kolmanovskii, V.B.: Stability in Viscoelasticity. North-Holland, Amsterdam (1994)

20. Lagnese, J.E., Lions, J.L.: In: Modelling, Analysis and Control of Thin Plates. Recherches en Mathématiques Appliquées, vol. 6. Mason, Paris (1988)

21. Prüss, J.: Evolutionary Integral Equations and Applications. Monographs in Mathematics, vol. 87. Birkhäuser, Basel (1993)

22. Keddi, A.A., Apalara, T.A., Messaoudi, S.A.: Exponential and polynomial decay in a thermoelastic-Bresse system with second sound. Appl. Math. Optim. 77(1), 315-341 (2018) 
23. Enyi, C.D., Mukiawa, S.E.: New general and explicit stability result for a thermoelastic Timoshenko system. Ric. Mat. (2021). https://doi.org/10.1007/s11587-021-00568-2

24. Dill, E.H.: Continuum Mechanics: Elasticity, Plasticity, Viscoelasticity. CRC Press, New York (2006)

25. Alves, D.M.O., Gomes Tavares, E.H., Silva, M.A.J., Rodrigues, J.H.: On modeling and uniform stability of a partially dissipative viscoelastic Timoshenko system. SIAM J. Math. Anal. 51(6), 4520-4543 (2019)

26. Mukiawa, S.E.: A new optimal and general stability result for a thermoelastic Bresse system with Maxwell-Cattaneo heat conduction. Results in Applied Mathematics 10 (2021). https://doi.org/10.1016/j.rinam.2021.100152

27. Mustafa, M.I.: General decay result for nonlinear viscoelastic equations. J. Math. Anal. Appl. 457, 134-152 (2018)

28. Arnold, V.I.: Mathematical Methods of Classical Mechanics. Springer, New York (1989)

29. Apalara, T.A.: General stability of memory-type thermoelastic Timoshenko beam acting on shear force. Contin. Mech. Thermodyn. 30, 291-300 (2018)

Submit your manuscript to a SpringerOpen ${ }^{\circ}$ journal and benefit from:

- Convenient online submission

$\checkmark$ Rigorous peer review

- Open access: articles freely available online

- High visibility within the field

- Retaining the copyright to your article

Submit your next manuscript at $\gg$ springeropen.com 